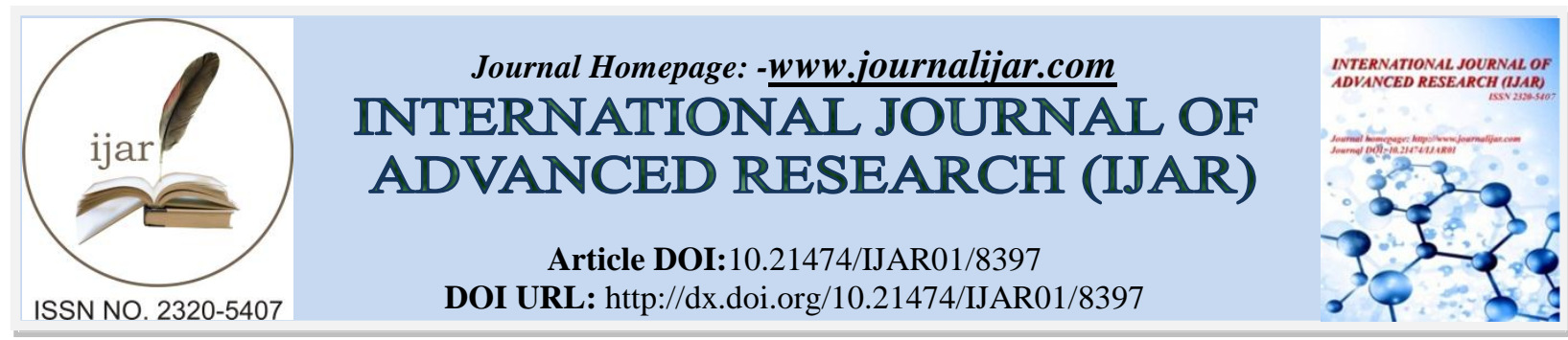

RESEARCH ARTICLE

\title{
THE EFFECT OF VITAMIN A ON EHRLICH ASCITES CARCINOMA GROWTH AND THE EXPRESSION OF P53, P16, TERT AND VEGFAS CANCER-RELATED GENES IN MICE.
}

\author{
Nesreen Ahmed Hassaneen ${ }^{1}$, Sabah M. Hassan ${ }^{1,2}$, Samar Rabah $^{1}$ and najlaSaud -Al saud ${ }^{1}$. \\ 1. Biology Department, Science College, king Abdulaziz University, Jeddah, Saudi Arabia. \\ 2. Department of Genetics, Faculty of Agriculture, Ain Shams University, Cairo, Egypt.
}

\section{Manuscript Info}

Manuscript History

Received: 12 November 2018

Final Accepted: 14 December 2018

Published: January 2019

\section{Abstract}

Background: In recent years, increasing number of studies demonstrated the effectiveness of vitamin A on preventing some types of cancer. Although; Vitamin A is well known as fat soluble vitamin with marked beneficial effects for human health, there is a contradictoryevidenceregard its safety in cancerous cases.

Purpose: Generally, excessive vitamin A levels had no preventive effect and in some cases were associated with increased cancer deaths. The proposed study aimed to highlight the importance of sufficient but not excessive intake of vitamins A by studding the potential preventing effect of taking vitamin A before the cancer illnesses and its harmful effect during the same illnesses.

Experimental Design: Healthy 45 male Swiss albino mice were used. They were randomly divided into five groups (each with 5 animals) group I was the tumor group (T) without any olive oil or vitamin A treatments as a control group, the other four groups weretreated with olive oil and the olive oil-resolved daily recommended dose of vitamin A $(0.2214 \mu \mathrm{l})$ using different plans. Solid tumor was induced in all groups by intramuscular injection of Ehrlich ascites carcinoma (EAC) cells $\left(2.5 \times 10^{6}\right.$ cells/ mice) in their right thighBody weight and tumor size were recorded at 0,2 ,3and 4 weeks and statistically analyzed. Expression levels of p53 and p16 genes studiesfor tumor tissuesweredone after 4 weeks.

Results: significant differences in body weight were found between control groups (T) and all the other groups. Administration of olive oil (O) and vitamin A before and after solid tumor inductionfound to beinsignificantly decreasedthe tumor circumferencescompared to untreated group $(\mathrm{T})$ with differences between the effects of treatments in each group. Vitamin A and olive oil found to have some effects on the expression levels of P16, P53, TERT and VEGFAgenes.

Conclusion: Administration of vitaminA by cancerous casesmust becritically controlled. In the current study, vitamin A daily supplementation found to be not effective in controlling EAC solid tumor growth as evidenced by both gross and gene expression examination.

Corresponding Author:-Nesreen Ahmed Hassaneen.

Address:-BiologyDepartment, Science College, king Abdulaziz University, Jeddah Saudi Arabia. 
Vitamin A supplementation must be avoided as it will act as prooxidant agents hindering the action of anticancer drugs.

Copy Right, IJAR, 2018,. All rights reserved.

\section{Introduction:-}

Cancer is a generic term for a large group of diseases that can affect any part of the body [1]. It is defined as an abnormal cell growth with tendency to invades or Spread to other parts of the body. Cancer ranks as the fourth leading cause of death in Mediterranean Region [2].

Abnormally high concentrations of free radicals in the body can be caused by exposure to ionizing radiation and other environmental toxins. The production of abnormally high levels of free radicals is the mechanism by which ionizing radiation work and resulted in tissue damage or cellular transformation [3].

Antioxidants are chemicals that interact with and neutralize free radicals, thus preventing them from causing damage and they are known asFree radical scavengers [3]. The body is usually makes its own antioxidants, which neutralize body free radicals. These antioxidants are called endogenous antioxidants. However, the body relies on external (exogenous) sources, primarily the diet, to obtain the rest of the antioxidants it needs. These exogenous antioxidants are commonly called dietary antioxidants. Fruits, vegetables and grains are rich sources of dietary antioxidants. Exogenous antioxidants are commonly worldwide taken as dietary supplements. Micronutrient such as vitamin A, C and $\mathrm{E}$ are reported to be potent antioxidant [3].

Vitamin A is the name of a group of fat-soluble retinoid, including retinol, retinal and retinyl esters .Vitamin A is involved in immune function, vision, reproduction and cellular communication $[4,5,6]$.

Botanical and natural dietary agents have been used for the treatment of cancer throughout history. These dietary agents also can be useful in the prevention of cancer. Epidemiological studies suggest that reduced risk of cancer is associated with high consumption of vegetables and fruits. The major part of total antioxidant activity is from the combination of photochemical [7].

Facts that must be taken in mind regarding some previous studies, which find out that, antioxidants accelerate the growth of some cancers [8]. A clinical study showed that supplementing the diet with the antioxidants $\mathrm{N}$ acetylcysteine (NAC) andvitamin E increases the tumor progression and reduces survival in mouse models of BRAF and K-RAS-induced lung cancer [9]. The authorsreported that, RNA sequencing revealed NAC and vitamin E, which are structurally unrelated, produce highly coordinated changes in tumor transcriptome profiles, dominated by reduced expression of endogenous antioxidant genes. NAC and vitamin E increase tumor cell by reducing ROS, DNA damage and P53.Antioxidants accelerate tumor growth by disrupting the ROS-p53 axis [9].

Smokers and other people at high risk for lung cancer could make it worse if they take antioxidant supplements when they developed lung cancer. Mice with lung cancer was found to develop more aggressive tumors and die faster when their diet is supplemented with antioxidants via deactivation of P53, the protein that neutralizing free radicals effect on tumors[10].

Several randomized controlled trials have investigated the effect of antioxidant supplements during cancer treatment and reported that they alters the effectiveness or reduce the toxicity of specific therapies and worsen the outcome of cancer especially in smoker persons [11].

In some preclinical studies, antioxidants have been found to promote tumor growth and metastasis in tumor-bearing mice and increase the ability of circulating tumor cells to metastasize [12]. Therefore, antioxidant supplements should be used with caution. Cancer patients should inform their doctors about their use of any dietary supplement.

\section{Research Objective:-}

Generally, excessive vitamin A levels had no preventive effect and in some cases were associated with increased cancer deaths. The proposed study aimed to highlight the importance of sufficient but not excessive intake of vitamins A by studding the potential preventing effect of taking vitamin A before the cancer illnesses and its harmful effect during the same illnesses. 


\section{Materials and Methods:- \\ Vitamin A:-}

Retinyl acetate powder was purchased from sigma chemicals, St Louis, USA. $0.2214 \mu \mathrm{g}$ has been dissolved in 100 $\mu \mathrm{l}$ olive oil and given to the treatment mice.

\section{Ehrlich Solid Tumor (EST):-}

Peritoneal fluid from Ehrlich bearing mice was collected and re-inoculated in the experimental animals and left for 7-10 days. The number of tumor cells per $\mathrm{ml}$ of ascetic fluid was determined by counting the cells with the help of hemocytometer. Ehrlich ascites carcinoma (EAC) fluid was injected in to mice intramuscularly in the right thigh in a dose of $0.2 \mathrm{ml}$ of ascetic fluid containing $2.5 \times 106$ cells. These cells were originally from American Type Tissue Culture Collection (Manassas, VA, USA).

\section{Animal model:-}

Healthy 25 male Swiss albino mice were obtained from King Fahd Medical Research Center, weighting 30 to $40 \mathrm{~g}$ and aged 8 to 10 weeks old. The animals were housed under conditions of controlled temperature (25-30c) with a $12 \mathrm{~h}$ day/night cycle. They were fed standard mouse pellet diet and given free access to water and wereacclimatized to laboratory condition for 10 days before commencement of the experiments.

\section{Tumor induction and treatments:-}

Animals were randomly divided into five groups (each with 5 animals), the used doses of olive oil and vitamin A were used according toAnroop\&Shery (2016) [13].

\section{Group I (T):-}

Injected withEAC cells $\left(0.2 \mathrm{ml}\right.$ of ascetic fluid containing $2.5 \times 10^{6}$ cells $)$ via intramuscular (IM) injection and observed for 14 days for tumor size (control group).

\section{Group II $(\mathbf{O}+\mathbf{T}+\mathbf{A}):-$}

Animals were orally received olive oil $(0.2214 \mu \mathrm{l})$ for 14 days, IM injected with $0.2 \mathrm{ml}$ EAC $\left(2.5 \times 10^{6}\right.$ cells $)$ and then theyorally received vitamin A $(0.2214 \mu \mathrm{l})$ for 14 days.

Group III (O+T+O):-

Animals were orally received olive oil $(0.2214 \mu \mathrm{l})$ for 14 days, IM injected with $0.2 \mathrm{ml} \mathrm{EAC}\left(2.5 \times 10^{6}\right.$ cells $)$ and then they orally received olive oil $(0.2214 \mu \mathrm{l})$ for 14 days.

\section{Group IV (A+T+A):-}

Animals were orally received vitamin A $(0.2214 \mu \mathrm{l})$ for 14 days, IM injected with $0.2 \mathrm{ml}$ EAC $(2.5 \times 106$ cells $)$ and then they orally received vitamin $\mathrm{A}(0.2214 \mu \mathrm{l})$ for 14 days.

\section{Group V (A+T+O):-}

Animals were orally received vitamin A $(0.2214 \mu \mathrm{l})$ for 14 days, IM injected with $0.2 \mathrm{ml} \mathrm{EAC}(2.5 \times 106$ cells $)$ and then they orally received olive oil $(0.2214 \mu \mathrm{l})$ for 14 days.

\section{Body weight measurements:-}

Body weights (BW) were taken four times; immediately before to EAC IM injection (zero time) and once a weekfor the nextthree weeks.

\section{Tumorcircumference measurements:-}

Tumor circumference wasmeasured twice a week for week one, two, three and four, using caliper throughout the experiments.

\section{Tumor growth inhibition rate (IR):-}

The tumor growth inhibition rate (IR) is calculated using the formula IR $(\%)=(1-$ AverageTWt/AverageTWc) $\mathrm{x}$ 100 , where TWt and TWc are the mean tumor weight of treated and control groups. 


\section{Gene expression analyses:-}

Total RNA was extracted from formalin fixed paraffin embedded blocks (FFPE) of solid Ehrlich tumor tissues by deparaffinization steps first and then we used the RNeasy FFPE Kit, following the manufacturer's instructions and reverse transcribed with quantitate reverse transcription kit, both from (QIAGEN). Real time reverse transcriptase PCR (qRT-PCR) was carried out by fast Sybr green master mix on step one plus. Synthesized cDNA was used as template for the amplification of P16, P53, TERT and VEGFA genes by (qRT-PCR). The housekeeping gene glyceraldehyde 3-phosphate dehydrogenase $(G A D P H)$ was used as an internal control gene to determine the effect of treatment strategies on the expression of P16, P53, TERT and VEGFAgenes. The relative expression of target genes wascomputed based on theirqRT-PCR [15].The sequences of the forward and reverse primes are shown in table (1).The qRT-PCR program was run as follows; at $95^{\circ} \mathrm{C}$ for 3 min (activation) and 40 cycles at $95^{\circ} \mathrm{C}$ for 15 $\sec , 58^{\circ} \mathrm{C}$ for $1 \mathrm{~min}$ (annealing) and $60^{\circ} \mathrm{C}$ for $1 \mathrm{~min}$ (extension). The results of these genes were normalized to GAPDH. Dissociation curves were also conducted after amplification to ensure the reaction specificity.

Table 1:-primer sequences forgapdh,p16, p53, tert and vegfa genes.

\begin{tabular}{|c|c|c|c|}
\hline Genes & Left primer & Right primer & Reference \\
\hline GAPDH & $\begin{array}{l}\text { 3'- } \\
\text { CAAGGTCATCCATGACAACTTTG- } \\
\text { 5' }\end{array}$ & $\begin{array}{l}\text { 3'- } \\
\text { GTCCACCACCCTGTTGCTGTAG- } \\
\text { 5' }\end{array}$ & [15] \\
\hline P16 & $\begin{array}{l}\text { 3'-TTGGCCCAAGAGCGGGGACA- } \\
5,\end{array}$ & $\begin{array}{l}\text { 3'- } \\
\text { GCGGGCTGAGGCCGGATTTA-5', }\end{array}$ & [15] \\
\hline P53 & 3'-TGCTCACCCTGGCTAAAGTT-5' & $\begin{array}{l}\text { 3'-AATGTCTCCTGGCTCAGAGG- } \\
\text { 5' }\end{array}$ & [15] \\
\hline TERT & $\begin{array}{l}3 \text { '-TCGAACAGCAAACCAACAGG- } \\
5 \text {, }\end{array}$ & $\begin{array}{l}\text { 3'-TCCACATCAGTTCCTGCAGT- } \\
\text { 5', }\end{array}$ & [16] \\
\hline VEGFA & $\begin{array}{l}\text { 3'-GCTGTAACGATGAAGCCCTG- } \\
\text { 5', }\end{array}$ & $\begin{array}{l}\text { 3'- } \\
\text { CGCTCCAGGATTTAAACCGG-5' }\end{array}$ & [16] \\
\hline
\end{tabular}

\section{Statistical Analysis:-}

The data were expressedas mean +/- standard error. The differences between the groups were calculated by SPSS windows Version 21 using Person correlation method the value of $(\mathrm{P}<0.05)$ was considered significant. The comparisons of gene expression between different experimental groups of mice were analyzed using $\Delta \Delta \mathrm{CT}$ method.

\section{Results and Discussion:-}

\section{Body Weight changes:-}

Within each group, $\mathrm{O}+\mathrm{T}+\mathrm{O}$ and $\mathrm{O}+\mathrm{T}+\mathrm{A}$ groups showed insignificant increase of the $\mathrm{BW}$ over the time, with significant $\mathrm{BW}$ increase before and after weeks of tumor inoculation in $\mathrm{A}+\mathrm{T}+\mathrm{O}$ and $\mathrm{A}+\mathrm{T}+\mathrm{A}$ groups. While, $\mathrm{O}+\mathrm{T}+\mathrm{O}$ group revealed insignificant reduction in the $\mathrm{BW}$ after one week of inoculation and it showed significant reduction in the BW after three weeks (Table 2). On the other hand, insignificant differenceswere observed between $\mathrm{T}$ group and the other groups regarding to in the initial, after seven days and after 14 days BW.While after 21 days of tumor inoculation, significant increase in the BW was observed in T group compared to all the other olive oil and vitamin A tumor treated groups (Table 3 and Figure 1).

Table 2:-Body weight changes during different weeks within the same mice group.

\begin{tabular}{|c|c|c|c|c|}
\hline $\begin{array}{r}\text { Group } \\
\text { Parameters } \\
\end{array}$ & $\begin{array}{c}\text { 1st week body } \\
\text { weight (grams) } \\
\text { at day } 0\end{array}$ & $\begin{array}{c}\text { 2nd week body } \\
\text { weight (grams) } \\
\text { at day } 7\end{array}$ & $\begin{array}{c}\text { 3rd week body } \\
\text { weight (grams) } \\
\text { at day } 14\end{array}$ & $\begin{array}{c}\text { Final body weight } \\
\text { (g) } \\
\text { at day } 21\end{array}$ \\
\hline $\mathrm{T}_{\text {group }}$ & $\begin{array}{c}30.57 \pm 0.06 \\
{ }_{\mathrm{b}}^{\mathrm{p}}=0.507 \\
{ }_{\mathrm{c}}^{\mathrm{p}}=0.312 \\
{ }_{\mathrm{d} p}^{\mathrm{d}}=0.028\end{array}$ & $\begin{array}{c}37.13 \pm 6.45 \\
{ }^{\mathrm{a}} \mathrm{p}=0.507 \\
{ }_{\mathrm{c} p}=0.973 \\
{ }_{\mathrm{d}}=0.209\end{array}$ & $\begin{array}{c}39.04 \pm 8.87 \\
{ }^{\mathrm{a}} \mathrm{p}=0.312 \\
{ }_{\mathrm{b}}=0.973 \\
{ }_{\mathrm{d}}=0.358\end{array}$ & $\begin{array}{r}47.00 \pm 1.74 \\
{ }^{\mathrm{a}} \mathrm{p}=0.028 \\
{ }_{\mathrm{b}}^{\mathrm{b}} \mathrm{p}=0.209 \\
{ }_{\mathrm{c}}^{\mathrm{c}} \mathrm{p}=0.358\end{array}$ \\
\hline & $33.50 \pm 1.93$ & $32.53 \pm 4.08$ & $34.10 \pm 7.10$ & $31.84 \pm 5.84$ \\
\hline
\end{tabular}




\begin{tabular}{|c|c|c|c|c|}
\hline $\mathrm{O}+\mathrm{T}+\mathrm{O}$ group & $\begin{array}{l}{ }^{b} p=0.995 \\
{ }^{c} p=0.999 \\
{ }^{d}=0.977\end{array}$ & $\begin{array}{r}{ }^{a} p=0.995 \\
{ }^{c} p=0.981 \\
{ }^{d} p=0.998\end{array}$ & $\begin{array}{l}{ }^{a} p=0.999 \\
{ }_{p} p=0.981 \\
{ }_{p}^{d}=0.946\end{array}$ & $\begin{array}{l}{ }^{a} p=0.977 \\
{ }_{p} p=0.998 \\
{ }^{c} p=0.946\end{array}$ \\
\hline $\mathrm{O}+\mathrm{T}+\mathrm{A}$ group & $\begin{array}{r}30.60 \pm 1.78 \\
{ }^{b} p=1.000 \\
{ }^{c} p=0.956 \\
{ }^{d} p=0.888\end{array}$ & $\begin{array}{r}30.53 \pm 1.96 \\
{ }^{\mathrm{a}} \mathrm{p}=1.000 \\
{ }_{\mathrm{c}}^{\mathrm{p}}=0.950 \\
{ }_{\mathrm{d}} \mathrm{p}=0.878\end{array}$ & $\begin{array}{r}32.11 \pm 5.28 \\
{ }^{a} p=0.956 \\
{ }_{p}^{b}=0.950 \\
{ }_{p}=0.996\end{array}$ & $\begin{array}{r}32.74 \pm 4.28 \\
{ }^{\mathrm{a}} \mathrm{p}=0.888 \\
{ }_{\mathrm{b}}=0.878 \\
{ }_{\mathrm{c}} \mathrm{p}=0.996\end{array}$ \\
\hline $\mathrm{A}+\mathrm{T}+\mathrm{O}$ group & $\begin{array}{r}31.74 \pm 2.19 \\
{ }_{\mathrm{b}} \mathrm{p}=0.944 \\
\mathrm{c} p=0.092 \\
{ }_{\mathrm{d}}=0.052\end{array}$ & $\begin{array}{r}33.12 \pm 4.46 \\
{ }^{\mathrm{a}} \mathrm{p}=0.944 \\
{ }^{\mathrm{c}} \mathrm{p}=0.198 \\
{ }_{\mathrm{d}}=0.113\end{array}$ & $\begin{array}{r}38.68 \pm 1.67 \\
{ }^{a} p=0.092 \\
{ }_{p} p=0.198 \\
{ }_{p}=0.976\end{array}$ & $\begin{array}{r}39.70 \pm 3.20 \\
{ }^{\mathrm{a}} \mathrm{p}=0.052 \\
{ }_{\mathrm{b}} \mathrm{p}=0.113 \\
{ }_{\mathrm{c}}^{\mathrm{p}}=0.976\end{array}$ \\
\hline $\mathrm{A}+\mathrm{T}+\mathrm{A}$ group & $\begin{array}{c}30.50 \pm 2.19 \\
{ }^{b} p=0.355 \\
{ }^{c} p=0.41 \\
{ }^{d} p=0.025\end{array}$ & $\begin{array}{c}33.61 \pm 2.19 \\
{ }_{\mathrm{a}}^{\mathrm{a}}=0.355 \\
\mathrm{c} p=0.439 \\
{ }_{\mathrm{d}} \mathrm{p}=0.285\end{array}$ & $\begin{array}{c}36.40 \pm 1.99 \\
{ }^{a} p=0.041 \\
{ }_{p} p=0.349 \\
d_{p}=0.983\end{array}$ & $\begin{array}{r}37.03 \pm 3.11 \\
{ }^{\mathrm{a}} \mathrm{p}=0.025 \\
{ }_{\mathrm{b}} \mathrm{p}=0.285 \\
{ }_{\mathrm{c}} \mathrm{p}=0.983\end{array}$ \\
\hline
\end{tabular}

Data expressed as mean \pm SE. values followed significance different from one another within thesame columns $(p \leq$ $0.05)$.

Table 3:-Body weight changes between mice groups that received different treatment plans of vitamin A and olive oil during the experiment

\begin{tabular}{|c|c|c|c|c|c|}
\hline Parameters & $\begin{array}{c}\mathbf{T} \\
\text { group }\end{array}$ & $\mathrm{O}+\mathrm{T}+\mathrm{O}$ group & $\mathrm{O}+\mathrm{T}+\mathrm{A}$ group & $\mathrm{A}+\mathrm{T}+\mathrm{O}$ group & $\begin{array}{c}\mathbf{A}+\mathbf{T}+\mathbf{A} \\
\text { Group }\end{array}$ \\
\hline $\begin{array}{c}\text { Body weight }(\mathrm{g}) \\
\text { at day } 0\end{array}$ & $\begin{array}{c}30.57 \pm 0.06 \\
{ }^{b} p=0.213 \\
{ }^{c} p=1.000 \\
{ }^{d} p=0.878 \\
{ }^{e} p=1.000\end{array}$ & $\begin{array}{c}33.50 \pm 1.93 \\
{ }^{a} p=0.213 \\
{ }^{c} p=0.222 \\
{ }^{d} p=0.644 \\
{ }^{e} p=0.197\end{array}$ & $\begin{array}{c}30.60 \pm 1.78 \\
{ }^{a} p=0.222 \\
{ }^{b} p=0.889 \\
{ }^{d} p=1.000 \\
{ }^{e} p=1.000\end{array}$ & $\begin{array}{c}30.50 \pm 0.52 \\
{ }^{a} p=0.644 \\
{ }^{b} p=0.889 \\
{ }^{c} p=0.855 \\
{ }^{e} p=0.878\end{array}$ & $\begin{array}{c}31.74 \pm 2.19 \\
{ }^{\mathrm{a}} \mathrm{p}=0.197 \\
\mathrm{~b} p=1.000 \\
{ }_{\mathrm{c}}^{\mathrm{c}}=0.855 \\
{ }_{\mathrm{d}} \mathrm{p}=1.000\end{array}$ \\
\hline $\begin{array}{c}\text { Body weight }(\mathrm{g}) \\
\text { at day } 7\end{array}$ & $\begin{array}{r}37.13 \pm 6.45 \\
{ }^{b} p=0.668 \\
{ }^{c} p=0.358 \\
{ }^{d} p=0.763 \\
{ }^{e} p=0.834\end{array}$ & $\begin{array}{c}32.53 \pm 4.08 \\
{ }^{a} p=0.974 \\
{ }^{c} p=1.000 \\
{ }^{d} p=0.997 \\
{ }^{e} p=0.668\end{array}$ & $\begin{array}{c}30.53 \pm 1.96 \\
{ }^{a} p=0.974 \\
{ }^{b} p=0.936 \\
{ }^{d} p=0.889 \\
{ }^{e} p=0.358\end{array}$ & $\begin{array}{c}33.12 \pm 4.46 \\
{ }^{a} p=1.000 \\
{ }^{b} p=0.936 \\
{ }^{c} p=1.000 \\
{ }^{e} p=0.763\end{array}$ & $\begin{array}{c}33.61 \pm 2.19 \\
{ }^{\mathrm{a}} \mathrm{p}=0.997 \\
{ }_{\mathrm{b}} \mathrm{p}=0.889 \\
{ }_{\mathrm{c}}^{\mathrm{p}}=1.000 \\
{ }_{\mathrm{d}}=0.834\end{array}$ \\
\hline $\begin{array}{c}\text { Body weight (g) } \\
\text { at day } 14\end{array}$ & $\begin{array}{r}39.04 \pm 8.87 \\
{ }^{b} p=0.824 \\
{ }^{c} p=0.594 \\
{ }^{d} p=1.000 \\
{ }^{e} p=0.977\end{array}$ & $\begin{array}{c}34.10 \pm 7.10 \\
{ }^{a} p=0.992 \\
{ }^{c} p=0.858 \\
{ }^{d} p=0.986 \\
{ }^{e} p=0.824\end{array}$ & $\begin{array}{c}32.11 \pm 5.28 \\
{ }_{\mathrm{a}} \mathrm{p}=0.992 \\
{ }_{\mathrm{b}} \mathrm{p}=0.637 \\
{ }_{\mathrm{d}} \mathrm{p}=0.883 \\
{ }_{\mathrm{e}} \mathrm{p}=0.594\end{array}$ & $\begin{array}{c}38.68 \pm 1.67 \\
{ }^{a} p=0.858 \\
{ }^{b} p=0.637 \\
{ }^{c} p=0.987 \\
{ }^{e} p=1.000\end{array}$ & $\begin{array}{c}36.40 \pm 1.99 \\
{ }^{a} p=0.986 \\
{ }^{b} p=0.883 \\
{ }^{c} p=0.987 \\
{ }_{p}=0.977\end{array}$ \\
\hline $\begin{array}{c}\text { Final body } \\
\text { weight (g) } \\
\text { at day } 21\end{array}$ & $\begin{array}{r}47.00 \pm 1.74 \\
{ }^{b} p=0.005 \\
{ }^{c} p=0.008\end{array}$ & $\begin{array}{c}31.84 \pm 5.84 \\
{ }_{\mathrm{a}}^{\mathrm{a}}=0.998 \\
{ }_{\mathrm{c}}=0.171\end{array}$ & $\begin{array}{c}32.74 \pm 4.28 \\
{ }_{\mathrm{a}}^{\mathrm{p}}=0.998 \\
{ }_{\mathrm{b}}=0.255\end{array}$ & $\begin{array}{c}39.70 \pm 3.20 \\
{ }^{\mathrm{a}} \mathrm{p}=0.171 \\
{ }_{\mathrm{b}} \mathrm{p}=0.255\end{array}$ & $\begin{array}{c}37.03 \pm 3.11 \\
{ }^{a} p=0.507 \\
{ }^{b} p=0.666\end{array}$ \\
\hline
\end{tabular}




\begin{tabular}{|l|l|l|l|l|l|}
\hline & ${ }^{\mathrm{d}} \mathrm{p}=0.220$ & ${ }^{\mathrm{d}} \mathrm{p}=0.507$ & ${ }^{\mathrm{d}} \mathrm{p}=0.666$ & ${ }^{\mathrm{c}} \mathrm{p}=0.911$ & ${ }^{\mathrm{c}} \mathrm{p}=0.911$ \\
& ${ }^{\mathrm{e}} \mathrm{p}=0.063$ & ${ }^{\mathrm{e}} \mathrm{p}=0.005$ & ${ }^{\mathrm{e}} \mathrm{p}=0.008$ & ${ }^{\mathrm{e}} \mathrm{p}=0.063$ & ${ }^{\mathrm{d}} \mathrm{p}=0.063$ \\
\end{tabular}

Data are expressed as mean $+/$ - SE. Significance versus $a(t), b(o+t+o), c(o+t+a), d(a+t+o), e(a+t+a) u s i n g$ Pearson's correlation $(\mathrm{p} \leq 0.05)$.

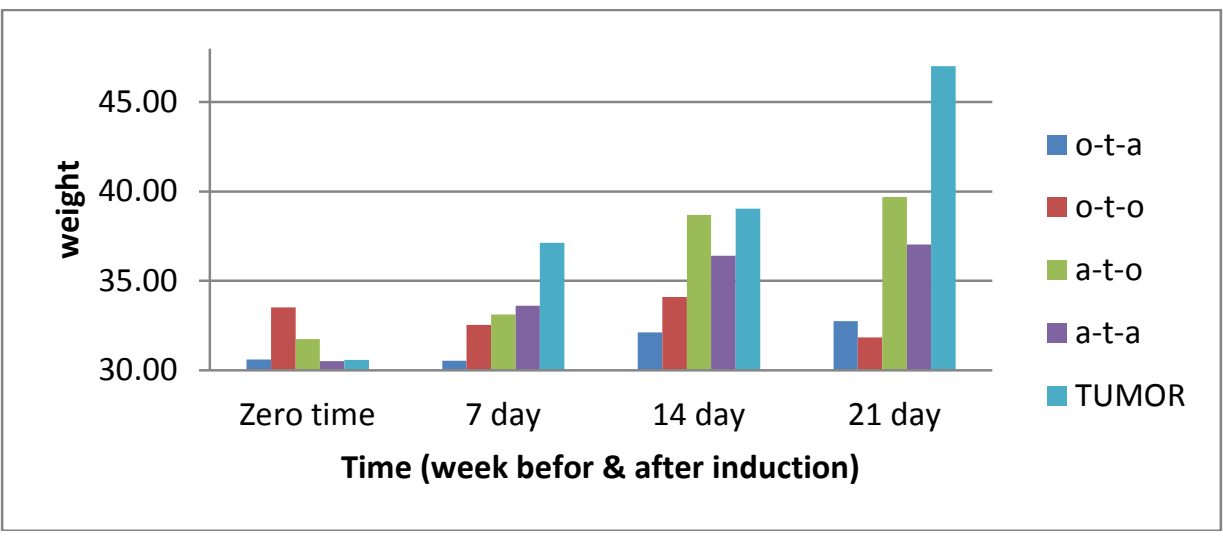

Figure 1:-Body weight changes between different mice groups that received different treatment plans of vitamin A and olive oil during the experiment.

Tumor circumference (TC) and growth inhibition:-

Table (4) and Figure (2) summarizes the results of the effect of vitamin A and olive oil administration on the solid tumor circumference (TC) after one, two, three and four weeks of inculcation. The TCs were increased in all experiment groups. Within each group, $\mathrm{T}$ group showed significant increase in the tumor circumference (TC)by the time. On the other hand some significantincreases in the TC were detected of the other groups. In $\mathrm{O}+\mathrm{T}+\mathrm{O}$ group significant increase in $\mathrm{TC}$ was observed after four weeks of inoculation compared to TC inthe same group after one week of inoculation. $\mathrm{O}+\mathrm{T}+\mathrm{A}$ group revealed significant differencesof the $\mathrm{TC}$ after one and two weeks compared to four weeks. $\mathrm{A}+\mathrm{T}+\mathrm{O}$ and $\mathrm{A}+\mathrm{T}+\mathrm{A}$ groups showed the most significant increases in $\mathrm{TC}$ after the $\mathrm{T}$ group.However, vitamin $\mathrm{A}$ and olive oiladministrations were ineffective in decreasing the TCs. The tumor growth inhibition rate reached $27.2 \%$ and $18.51 \%$ for $\mathrm{O}+\mathrm{T}+\mathrm{O}, \mathrm{O}+\mathrm{T}+$ Agroups as the maximum values, whereas, the rate of $\mathrm{A}+\mathrm{T}+$ Ogroupreached $10.477 \%$, theminimum value of tumor growth inhibition rate was $7.154 \%$ in $\mathrm{A}+\mathrm{T}+\mathrm{A}$ group (Table 5).

Table 4:-Tumor circumference during different weeks within the same mice group.

\begin{tabular}{|c|c|c|c|c|}
\hline $\begin{array}{l}\text { Parameters } \\
\text { Group } \\
\end{array}$ & $\begin{array}{c}\text { Tumor } \\
\text { circumference }(\mathrm{mm}) \\
\text { after 7days of } \\
\text { inculcation }\end{array}$ & $\begin{array}{c}\text { Tumor } \\
\text { circumference }(\mathrm{mm}) \\
\text { after } 14 \text { days of } \\
\text { inculcation }\end{array}$ & $\begin{array}{c}\text { Tumor } \\
\text { circumference }(\mathrm{mm}) \\
\text { after } 21 \text { days of } \\
\text { inculcation }\end{array}$ & $\begin{array}{c}\text { Tumor } \\
\text { circumference }(\mathrm{mm}) \\
\text { after } 28 \text { days of } \\
\text { inculcation }\end{array}$ \\
\hline $\begin{array}{l}\text { T } \\
\text { group }\end{array}$ & $\begin{array}{c}7 \pm 67 \\
{ }_{p} p=0.005 \\
{ }^{c} p=0.00 \\
{ }_{p} p=0.00\end{array}$ & $\begin{array}{l}12.67 \pm 0.58 \\
{ }_{\mathrm{a}}^{\mathrm{p}}=0.005 \\
{ }_{\mathrm{c}}^{\mathrm{p}}=0.118 \\
{ }_{\mathrm{d}}^{\mathrm{p}}=0.003\end{array}$ & $\begin{array}{c}15.33 \pm 0.58 \\
{ }^{a} p=0.00 \\
{ }^{b} p=0.118 \\
{ }^{d} p=0.074\end{array}$ & $\begin{array}{c}18.33 \pm 2.31 \\
{ }^{a} \mathrm{p}=0.00 \\
{ }_{\mathrm{b}} \mathrm{p}=0.003 \\
{ }_{\mathrm{c}} \mathrm{p}=0.074\end{array}$ \\
\hline $\mathrm{O}+\mathrm{T}+\mathrm{O}$ group & $\begin{array}{l}6.33 \pm 0.58 \\
{ }_{\mathrm{b}}=0.653 \\
\mathrm{c}_{\mathrm{p}}=0.653\end{array}$ & $\begin{array}{l}8.67 \pm 1.15 \\
{ }_{\mathrm{a}} \mathrm{p}=0.653 \\
\mathrm{c}_{\mathrm{p}}=1.000\end{array}$ & $\begin{array}{l}8.67 \pm 1.15 \\
{ }_{\mathrm{a}}^{\mathrm{a}}=0.653 \\
{ }_{\mathrm{b}}=1.000\end{array}$ & $\begin{array}{c}12.67 \pm 4.51 \\
{ }_{\mathrm{a}}^{\mathrm{a}}=0.049 \\
{ }_{\mathrm{b}} \mathrm{p}=0.255\end{array}$ \\
\hline
\end{tabular}




\begin{tabular}{|c|c|c|c|c|}
\hline & ${ }^{\mathrm{d}} \mathrm{p}=0.049$ & ${ }^{d} \mathrm{p}=0.255$ & ${ }^{d} p=0.255$ & ${ }^{c} \mathrm{p}=0.255$ \\
\hline $\mathrm{O}+\mathrm{T}+\mathrm{A}$ group & $\begin{array}{l}6.33 \pm 0.58 \\
{ }_{p}=0.376 \\
{ }^{c} p=0.002 \\
{ }_{p} p=0.000\end{array}$ & $\begin{array}{l}8.00 \pm 0.00 \\
{ }^{a} p=0.376 \\
{ }^{c} p=0.014 \\
{ }_{p}^{d}=0.0001\end{array}$ & $\begin{array}{c}12.00 \pm 0.00 \\
{ }^{a} p=0.002 \\
{ }_{p}^{b}=0.014 \\
{ }^{d} p=0.154\end{array}$ & $\begin{array}{c}14.33 \pm 2.31 \\
{ }^{\mathrm{a}} \mathrm{p}=0.000 \\
{ }_{\mathrm{b}}^{\mathrm{b}}=0.001 \\
{ }_{\mathrm{c}}^{\mathrm{c}}=0.154\end{array}$ \\
\hline $\mathbf{A}+\mathbf{T}+\mathbf{O}$ group & $\begin{array}{l}7.33 \pm 0.58 \\
{ }_{\mathrm{b}} \mathrm{p}=0.033 \\
{ }_{\mathrm{c} p}^{\mathrm{p}}=0.012 \\
{ }_{\mathrm{d}} \mathrm{p}=0.001\end{array}$ & $\begin{array}{r}12 \pm 1.73 \\
{ }^{a} p=0.033 \\
{ }_{p} p=0.874 \\
{ }^{d} p=0.095\end{array}$ & $\begin{array}{r}13.00 \pm 1.73 \\
{ }^{\mathrm{a}} \mathrm{p}=0.012 \\
{ }^{\mathrm{b}} \mathrm{p}=0.874 \\
{ }_{\mathrm{d}}^{\mathrm{d}}=0.264\end{array}$ & $\begin{array}{c}15.67 \pm 2.08 \\
{ }^{\mathrm{a}} \mathrm{p}=0.001 \\
{ }_{\mathrm{b}}^{\mathrm{b}}=0.095 \\
{ }_{\mathrm{c}}^{\mathrm{c}}=0.0264\end{array}$ \\
\hline $\mathbf{A}+\mathbf{T}+\mathbf{A}$ group & $\begin{array}{l}7.67 \pm 0.58 \\
{ }_{p} p=0.336 \\
{ }_{p} p=0.028 \\
{ }_{p}=0.000\end{array}$ & $\begin{array}{c}12.67 \pm 0.58 \\
{ }_{\mathrm{a}}^{\mathrm{p}}=0.336 \\
\mathrm{c} p=0.336 \\
{ }_{\mathrm{d}}=0.002\end{array}$ & $\begin{array}{c}15.33 \pm 0.58 \\
{ }^{\mathrm{a} p}=0.028 \\
{ }_{\mathrm{b}}=0.336 \\
{ }_{\mathrm{d}}=0.019\end{array}$ & $\begin{array}{c}18.33 \pm 2.31 \\
{ }^{a} p=0.00 \\
{ }_{p}^{b}=0.002 \\
{ }^{c} p=0.019\end{array}$ \\
\hline
\end{tabular}

Data are expressed as mean +/- SE. significance different from one another within a (TCF in wk1),b (TCF in wk2),c (TCF in wk3),d (TCF in wk4) using Pearson's correlation ( $\mathrm{p} \leq 0.05)$.

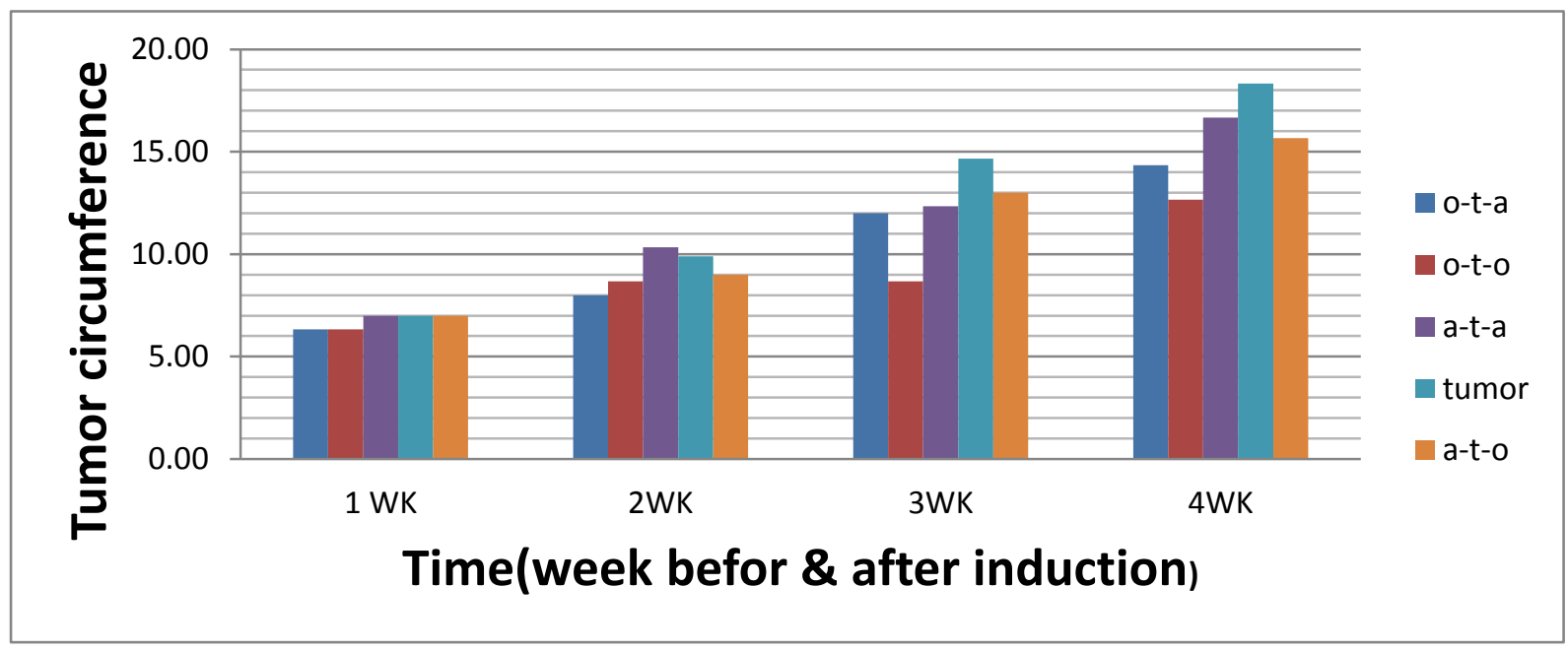

Figure 2:-Tumor circumference in different mice groups that received different treatment plans of vitamin $\mathrm{A}$ and olive oil during the experiment.

Gene expression on tumor tissue:-

Total RNA was extracted from solid tumors of each mice and the expression levels of P16, P53, TERT and VEGFA genes were examined by qRT-PCR method as presented in Table (6) and figure(3). Results showed that the expression of $\mathrm{P} 16$ was upregulated in $\mathrm{A}+\mathrm{T}+\mathrm{O}, \mathrm{O}+\mathrm{T}+\mathrm{O}$ and $\mathrm{A}+\mathrm{T}+\mathrm{A}$ groups, while it was downregulated in $\mathrm{O}+\mathrm{T}+\mathrm{A}$ group compared to untreated tumor ( $\mathrm{T}$ ) group. On the other hand, the expression level of P53 was upregulated in $\mathrm{O}+\mathrm{T}+\mathrm{O}, \mathrm{A}+\mathrm{T}+\mathrm{A}, \mathrm{O}+\mathrm{T}+\mathrm{A}$ and $\mathrm{A}+\mathrm{T}+\mathrm{O}$ groups compared to $\mathrm{T}$ group. For TERT gene the expression levels were upregulated in $\mathrm{A}+\mathrm{T}+\mathrm{O}$ group, while they were downregulated in $\mathrm{A}+\mathrm{T}+\mathrm{A}, \mathrm{O}+\mathrm{T}+\mathrm{O}$ and $\mathrm{O}+\mathrm{T}+\mathrm{A}$ compared to $\mathrm{T}$ group. VEGFA showed upregulation in $\mathrm{O}+\mathrm{T}+\mathrm{O}, \mathrm{O}+\mathrm{T}+\mathrm{A}$ and $\mathrm{A}+\mathrm{T}+\mathrm{A}$ groups, whereas it showed downregulation in $\mathrm{A}+\mathrm{T}+\mathrm{O}$ group compared to $\mathrm{T}$ group. 
Table 5:-Tumor circumference and inhibition rate in different mice groups that received different treatment plans of vitamin A and olive oil during the experiment.

\begin{tabular}{|c|c|c|c|c|c|}
\hline Parameters & $\begin{array}{l}\mathbf{O + T}+\mathbf{A} \\
\text { group }\end{array}$ & $\mathrm{O}+\mathrm{T}+\mathrm{O}$ group & $\mathbf{A}+\mathbf{T}+\mathbf{O}$ group & $\mathbf{A}+\mathbf{T}+\mathbf{A}$ group & $\begin{array}{c}\text { T } \\
\text { Group }\end{array}$ \\
\hline $\begin{array}{c}\text { Tumor } \\
\text { circumference }(\mathbf{m m}) \\
\text { after } 1 \text { wk of } \\
\text { inculcation }\end{array}$ & $\begin{array}{l}6.33 \pm 0.58 \\
{ }^{b} \mathrm{P}=1.000 \\
{ }^{\mathrm{c}} \mathrm{P}=0.283 \\
{ }^{\mathrm{d}} \mathrm{P}=0.012 \\
{ }^{\mathrm{e}} \mathrm{P}=0.102\end{array}$ & $\begin{array}{l}6.33 \pm 0.58 \\
{ }^{\mathrm{a}} \mathrm{P}=1.000 \\
{ }^{\mathrm{c}} \mathrm{P}=0.283 \\
{ }^{\mathrm{d}} \mathrm{P}=0.012 \\
{ }^{\mathrm{e}} \mathrm{P}=0.102\end{array}$ & $\begin{array}{c}7 \pm 0.00 \\
{ }^{\mathrm{a}} \mathrm{P}=0.283 \\
{ }^{\mathrm{b}} \mathrm{P}=0.283 \\
{ }^{\mathrm{d}} \mathrm{P}=0.283 \\
{ }^{\mathrm{e}} \mathrm{P}=0.950\end{array}$ & $\begin{array}{c}7 \pm 0.00 \\
{ }^{\mathrm{a}} \mathrm{P}=0.012 \\
{ }^{\mathrm{b}} \mathrm{P}=0.012 \\
{ }^{\mathrm{c}} \mathrm{P}=0.283 \\
{ }^{\mathrm{e}} \mathrm{P}=0.633\end{array}$ & $\begin{array}{c}7 \pm 0.00 \\
{ }^{\mathrm{a}} \mathrm{P}=0.102 \\
{ }^{\mathrm{b}} \mathrm{P}=0.102 \\
{ }^{\mathrm{c}} \mathrm{P}=0.950 \\
{ }^{\mathrm{d}} \mathrm{P}=0.633\end{array}$ \\
\hline $\begin{array}{c}\text { Tumor } \\
\text { circumference }(\mathbf{m m}) \\
\text { after } 2 \text { wk of } \\
\text { inculcation }\end{array}$ & $\begin{array}{l}8 \pm 0.00 \\
{ }^{\mathrm{b}} \mathrm{P}=0.00 \\
{ }^{\mathrm{c}} \mathrm{P}=0.00 \\
{ }^{\mathrm{d}} \mathrm{P}=0.00 \\
{ }^{\mathrm{e}} \mathrm{P}=0.00\end{array}$ & $\begin{array}{l}8.67 \pm 1.15 \\
{ }^{\mathrm{a}} \mathrm{P}=0.920 \\
{ }^{\mathrm{c}} \mathrm{P}=0.015 \\
{ }^{\mathrm{d}} \mathrm{P}=0.314 \\
{ }^{\mathrm{e}} \mathrm{P}=0.004\end{array}$ & $\begin{array}{c}9 \pm 0.00 \\
{ }^{\mathrm{a}} \mathrm{P}=0.015 \\
{ }^{\mathrm{b}} \mathrm{P}=0.004 \\
{ }^{\mathrm{d}} \mathrm{P}=0.314 \\
{ }^{\mathrm{e}} \mathrm{P}=0.920\end{array}$ & $\begin{array}{c}10.33 \pm 0.58 \\
{ }^{\mathrm{a}} \mathrm{P}=0.314 \\
{ }^{\mathrm{b}} \mathrm{P}=0.098 \\
{ }^{\mathrm{c}} \mathrm{P}=0.314 \\
{ }^{\mathrm{e}} \mathrm{P}=0.098\end{array}$ & $\begin{array}{l}9.9 \pm 1.68 \\
{ }^{\mathrm{a}} \mathrm{P}=0.004 \\
{ }^{\mathrm{b}} \mathrm{P}=0.001 \\
{ }^{\mathrm{c}} \mathrm{P}=0.920 \\
{ }^{\mathrm{d}} \mathrm{P}=0.098\end{array}$ \\
\hline $\begin{array}{c}\text { Tumor } \\
\text { circumference }(\mathbf{m m}) \\
\text { after } 3 \text { wk of } \\
\text { inculcation }\end{array}$ & $\begin{array}{l}12.00 \pm 0.00 \\
{ }^{b} \mathrm{P}=0.015 \\
{ }^{\mathrm{c}} \mathrm{P}=0.738 \\
{ }^{\mathrm{d}} \mathrm{P}=0.993 \\
{ }^{\mathrm{e}} \mathrm{P}=0.015\end{array}$ & 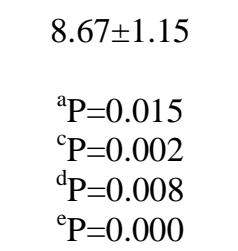 & $\begin{array}{l}13 \pm 1.73 \\
{ }^{\mathrm{a}} \mathrm{P}=0.002 \\
{ }^{\mathrm{b}} \mathrm{P}=0.738 \\
{ }^{\mathrm{d}} \mathrm{P}=0.920 \\
{ }^{\mathrm{e}} \mathrm{P}=0.098\end{array}$ & $\begin{array}{c}12.33 \pm 0.58 \\
{ }^{a} \mathrm{P}=0.008 \\
{ }^{\mathrm{b}} \mathrm{P}=0.993 \\
{ }^{\mathrm{c}} \mathrm{P}=0.920 \\
{ }^{\mathrm{e}} \mathrm{P}=0.028\end{array}$ & $\begin{array}{l}14.67 \pm 0.58 \\
{ }^{a} P=0.000 \\
{ }^{b} P=0.015 \\
{ }^{c} P=0.098 \\
{ }^{d} P=0.028\end{array}$ \\
\hline $\begin{array}{c}\text { Tumor } \\
\text { circumference }(\mathrm{mm}) \\
\text { after } 4 \text { wk of } \\
\text { inculcation }\end{array}$ & $\begin{array}{l}14.33 \pm 2.31 \\
{ }^{b} \mathrm{P}=0.950 \\
{ }^{\mathrm{c}} \mathrm{P}=0.977 \\
{ }^{\mathrm{d}} \mathrm{P}=0.854 \\
{ }^{\mathrm{e}} \mathrm{P}=0.477\end{array}$ & 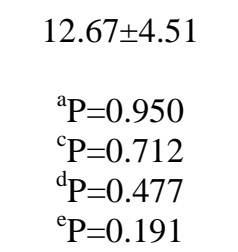 & $\begin{array}{l}15.67 \pm 2.08 \\
{ }^{\mathrm{a}} \mathrm{P}=0.712 \\
{ }^{\mathrm{b}} \mathrm{P}=0.977 \\
{ }^{\mathrm{d}} \mathrm{P}=0.992 \\
{ }^{\mathrm{e}} \mathrm{P}=0.787\end{array}$ & $\begin{array}{l}16.67 \pm 2.52 \\
{ }^{a} \mathrm{P}=0.477 \\
{ }^{\mathrm{b}} \mathrm{P}=0.854 \\
{ }^{\mathrm{c}} \mathrm{P}=0.992 \\
{ }^{\mathrm{e}} \mathrm{P}=0.950\end{array}$ & $\begin{array}{l}18.33 \pm 2.31 \\
{ }^{\mathrm{a}} \mathrm{P}=0.191 \\
{ }^{\mathrm{b}} \mathrm{P}=0.477 \\
{ }^{\mathrm{c}} \mathrm{P}=0.787 \\
{ }^{\mathrm{d}} \mathrm{P}=0.950\end{array}$ \\
\hline $\begin{array}{l}\text { Average tumor } \\
\text { circumference }\end{array}$ & 10.165 & 9.085 & 11.168 & 11.583 & 12.475 \\
\hline $\begin{array}{c}\text { tumor growth } \\
\text { inhibition rate (IR) } \\
\%\end{array}$ & $18.51 \%$ & $27.2 \%$ & $10.477 \%$ & $7.154 \%$ & 0.00 \\
\hline
\end{tabular}

Data expressed as mean \pm SE .Significance versus $a(0+t+a), b(0+t+o), c(a+t+o), d(a+t+a), e(t) u s i n g$ Pearson's correlation $(\mathrm{p} \leq 0.05)$.

Table 6:- Relative expression of target genes in each group in comparison to reference gene, $\mathrm{RE}=2[\Delta \mathrm{CT}]=2[\mathrm{CT}$ reference gene -CT target gene]

\begin{tabular}{|c|c|c|c|c|}
\hline Groups & \multicolumn{4}{|c|}{ Genes (Relative Expression) } \\
\hline & P16 & P53 & TERT & VEGF \\
\hline $\mathrm{O}+\mathrm{T}+\mathrm{A}$ & 0.00119 & 0.00285 & 0.00216 & 0.00567 \\
\hline $\mathrm{O}+\mathrm{T}+\mathrm{O}$ & 0.00792 & 0.00632 & 0.00306 & 0.00843 \\
\hline $\mathrm{A}+\mathrm{T}+\mathrm{A}$ & 0.00616 & 0.00317 & 0.00148 & 0.00251 \\
\hline $\mathrm{A}+\mathrm{T}+\mathrm{O}$ & 0.00866 & 0.00198 & 0.00932 & 0.00119 \\
\hline $\mathrm{T}$ & 0.00139 & 0.00125 & 0.00373 & 0.00221 \\
\hline
\end{tabular}



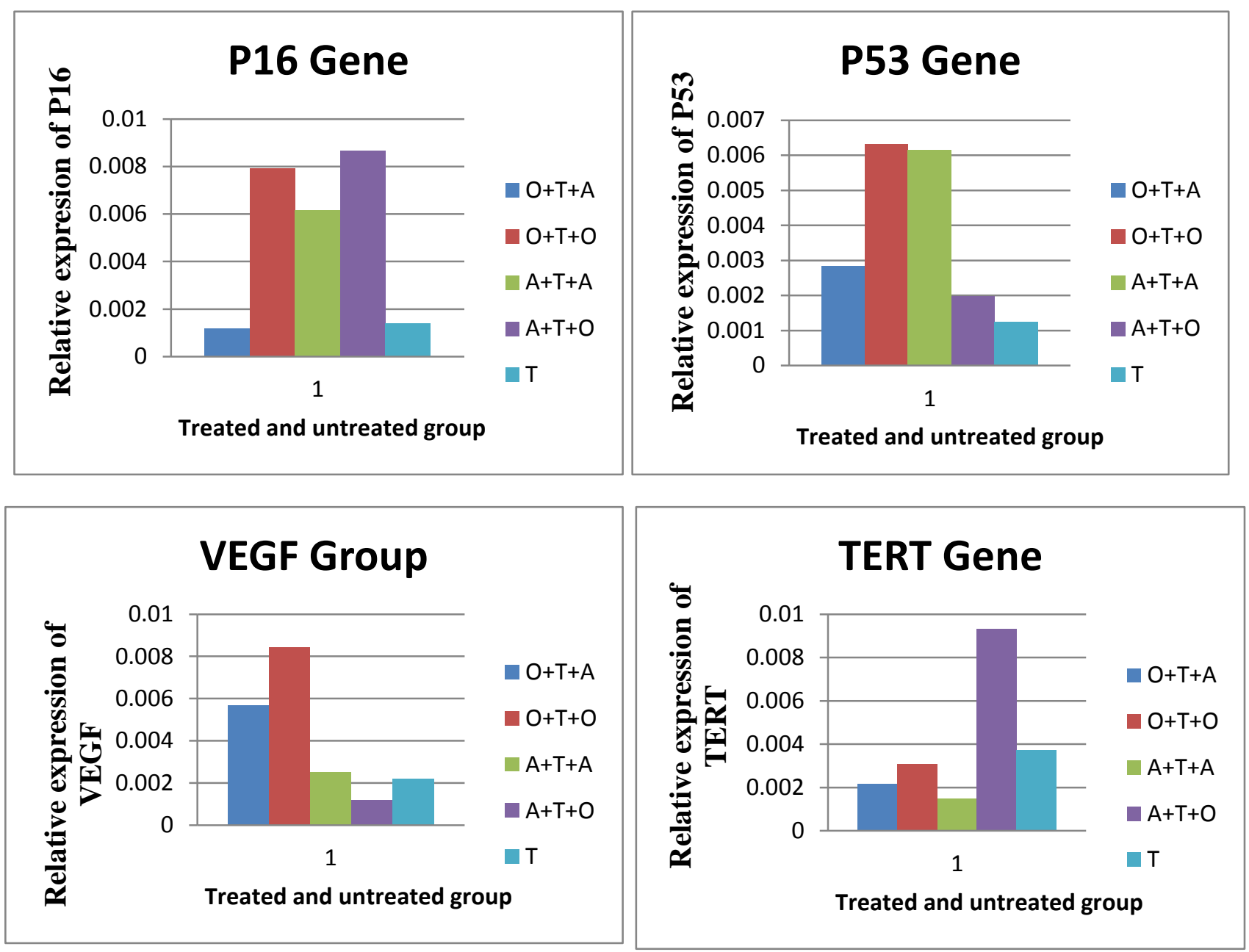

Figure 3:-Effect of different treatment on the expiration of P16, P53, TERT, VEGF, genesin EAC using qRT-PCR analysis.

\section{Discussion:-}

Dietary supplements such as vitamins and minerals have become widely available to be taken for a variety of reasons. Some people are advised by their doctors or dietitian to take dietary supplements especially if they have certain medical conditions such as pregnancy or those who have a restricted dietary intake. Many people also take vitamins and supplements while they are being treated for cancer; others take them to prevent cancer.Cancer patients are increasingly turning to supplements to strengthen their immune systems and ease nausea from chemotherapy [15].Until now, the role of vitamin supplements in preventing breast cancer remains unclear.Contradictory data regard beneficial versus unwanted potentiating carcinogenic effect of vitamin A. Epidemiological studies have suggested an inverse correlation between cancer development and dietary consumption of vitamin A.Our results indicated that vitamin A administration before and after the injection of the EAC cells increased, the BW and TC, these results are in disagreement with a study who suggested that the pharmacological concentrations of vitamin A decrease the incidence of chemically induced experimental tumors [16]. Also other studies reported that natural and synthetic retinoid have been demonstrated to inhibit the growth and the development of different types of tumors, including skin, breast, oral cavity, lung, hepatic, gastrointestinal, prostatic, and bladder cancers [17-18]. Clinical evidencesshows that vitamin A helps to prevent cancer. The association between vitamin A and cancer was initially reported in 1926 when rats, fed vitamin A-deficient diet, developed gastric carcinomas. The first investigation showing a relationship between vitamin A and human cancer was performed in 1941 by Abelsetal who found low plasma vitamin A levels in patients with gastrointestinal cancer [19]. Moon et al. reported that daily supplemental 
doses of 25,000 IU of vitamin A prevented squamous cell carcinoma. Studies that use animal models have shown that retinoids (including vitamin A) can act in the promotion-progression phase of carcinogenesis and block the development of invasive carcinoma at several epithelial sites, including the head and neck and lung. [19].Vitamin A supplementation likely does not reduce chemotherapy efficacy, and for many patients, is unlikely to cause harm. [20].

Vitamin Awas advised by cliniciansfor its antioxidant activity once thought to help preventing or promotingcancertherapy. However, our results are in agreement with more recent studies, which showed that high levels of these supplement may increase cases of gastrointestinal cancers, such as stomach, colon, and esophageal, in some populations. [15]

In the current study, although there were no significant decrease in the final CT between the olive oil and vitamin treated groups and tumor untreated group,P16and P53 expression levels were increased with olive oil and vitamin A treated groups (decreased TC) compared to tumor untreated group (maximum TC). The results of the study have demonstrated that treatment by vitamin A and olive oil has no prevention or productive effect. These results are in agreement with [15] who reported that P16 and P53have important roles in the inhibition of cell proliferation via cell cycle arrest and apoptosis. They are mutated in many tumor cell types [16].Human telomerase reverse transcriptase TERT, which regulates telomere length and can promote tumor development, increases the anti-apoptotic capacity of cells and enhance repair. It is thought to aid tumor cell growth and allow tumor cells to become immortal, so that TERT expression is considered a potential target for anticancer therapy.In our study, expression levels of TERTwereelevated in tumor tissue for treated group with vitamin A before inculcated with solid tumor in comparison with non-treated group. $V E G V$ is known as vascular endothelial growth factor (formation of new blood vessels) in this study the expression level of this gene found to be high in $\mathrm{O}+\mathrm{T}+\mathrm{O}$ group, most tumors show higher levels of $V E G F$, higher levels mean low chance of survival.

In the present study we investigate the potential effect of vitamin A on EAC cell development, which is inoculated in mice thigh to develop solid tumors. Recommended dose in literature was reported to be ranged from (900 mcg RAE for male- 700 mcg RAE for female) [21].

\section{Conclusion:-}

while dietary factors may be crucial in modifying cancer risk, the role of vitamin supplements in preventing cancer still remains unclear.In the present study, we examined the effects of vitamin A supplement in mice with solid tumor.Our data did not demonstrate any significant effect of vitamin A supplementation on neither decreasing or increasing of the TCbut it has some effects on the expression levels of P16, P53, TERT and VEGFAgenes. All animal treated groups didn't show any significant reduction in TC compered to untreated group (T) and the average volume of tumors were similar. Further investigations are warranted to elucidate the mechanisms by which vitamin A supplementation may modify cancer development.

\section{References:-}

1. Parkin, M., B, L. \& Walker, L., (2011). The fraction of cancer attributabletolifestyle andenvironmental factors in the UK. British Journal of Cancer.105Suppl 2: S77-81.

2. Ferlay, J. Soerjomataram, I. Ervik, M. Dikshit, R. Eser, S \& Mathers, C,(2012). CancerIncidence and Mortality Worldwide: IARC Cancer Base No. 11.

3. Lobo, V. Patil, A. Phatak, A. \& Chandra, N, (2010) .Free radicals, antioxidantsandfunctionalfoods: Impact on human health. Pharmacogn Rev, 4(8): 118-126.

4. Vitamin A, (2015). Micronutrient Information Center, Linus Pauling Institute, Oregon state university.

5. Vitamin A, (2016). MedlinePlus, National Library of Medicine, US National InstituteofHealth.

6. Vitamin A, (2011). Biomarkers of nutrition for development. The American Journal of Clinical Nutrition.

7. Norleena, P.Gullett, A. Ruhul, A, B. Bayraktar, C. John, M. Pezzuto, D.Dong ,B. Fadlo, R;Khuri, B. Bharat, B,Aggarwal, E,Young-JoonSurh F \& Omer Kucuk, B.,(2010). Cancer prevention with natural compounds. Seminars in Oncology, Volume 37, Issue 3, Pages 258-281.

8. Singh.P.P.Chandra, ANU; Farzana, M, Roy, A \&Sharma, P., (2010).Reconvene and Reconnect the Antioxidant Hypothesis in Human Health and Disease. Indian J ClinBiochem

9. Sayin, I. Ibrahim, M. Larsson, E.Nilsson, J. Lindah, P. \&Bergo, M., (2014). Antioxidants accelerate lung cancer progression in mice, Science translational Medicine 9; 6(221):221ra15. 
10. Bergo, M.Lindahl, P \& Campbell, P., (2014). Could antioxidants speed up Cancer rogression.American cancer society, Science translational medicine.

11. Asuka, Y. Hayato, U \&Toshinori, Ito. (2016). Efficacy and Interaction of Antioxidant Supplements as Adjuvant Therapy in Cancer Treatment. Integr Cancer Ther. 15(1): 17-39.

12. Thyagarajan, A \&Sahu, R, P., (2018). Potential Contributions of Antioxidants to Cancer Therapy: Immunomodulation and Radio sensitization. Integrative Cancer Therapies. 17(2): 210-216.

13. Anroop, B, N\&Shery, J., (2016). A simple practice guide for dose conversion between animals and human .Journal of basic and clinical pharmacy .7(2):27-31.

14. Quantitect SYBR green RT-PCR hand book (2011) .for quantitative, real time one step RT-PCR using SYBR green 1, qiagen.

15. Bassiony, H.Sabet, S .El-Din, T.Mohamed, M.El-Ghor, A, (2014). Magnetite nanoparticles inhibit tumorgrowth and upregulated the expression of p53/p16 in Ehrlich solid carcinoma bearing mice.

16. Mikirova, N.Scimeca, R, (2016).Gene expression response to ascorbic acid in mice implanted with sarcoma S180 cells.

17. Arrieta, O. González-De La Rosa, H. \&Aréchaga-Ocampo E. (2010) Randomized phase II trial of All-transretinoic acid with chemotherapy Based on paclitaxel and cisplatin as first-line treatment in patients with Advanced non-small-cell lung cancer. Journal of Clinical Oncology.

18. Bryan, M. Pulte , D\& Toomey , C.(2011) A pilot phase II trial of all- Tran's retinoic acid (Vesanoid) and paclitaxel (Taxol) in patients withRecurrent or metastatic breast cancer. Investigational new drugs.

19. www.bccancer.bc.ca/PPI/UnconventionalTherapies/VitaminARetinol.htm

20. Andrea, S. \& Blevins, P.(2017) Vitamin A and Cancer. Cancer Therapy Advisor

21. Areida, S.Abd el-azim, O\&Amer, E. (2015Protective and curative effect of thymoquinone on Ehrlich solid carcinoma inoculated mice .The egyption journal of hospital medicine. 